\title{
Strategic management of the integrated design of product and production system
}

\author{
Agostino Villa \\ Dipartimento di Sistemi di Produzione ed Economia dell'Azienda \\ Politecnico di Torino, c.so Duca degli Abruzzi, 24 I-10129 Torino \\ Phone $n^{\circ}+39-11-564-7233$; fax: +39-11-564-7299; \\ e-mail address: villa@athena.polito.it
}

\begin{abstract}
A process for designing a new final product and the related manufacturing system is really complex and time-consuming owing to its combinatorial nature. This paper outlines a general design methodology based on a decomposition of the design problem according to the following main line: a design has to be developed, by using models of greater and greater detail (progressive innovation). The paper will outline a new iterative design approach, based on an "innovation loop" which progressively impoves the design results.
\end{abstract}

\section{Keywords}

Industrial design, iterative innovation, decisinal task management.

\section{INTRODUCTION}

The goal of the design of a new product and related production system is the definition of a procedure for driving how to build the system itself in such a way that it could perform according to a-priori stated structural requirements and functional specifications (design target). Said procedure is usually called "design 
manual", that means the organized set of the prescriptions which drive industrial operations required for producing the new final product and for organizing the innovated production system. The decisional process which aims at defining a design manual is referred to as the "design process": this one is the specific topics of our interest.

Technical literature presents two complementary approaches to the organization of industrial design process (see Villa, 1997 and Villa et al., 1996 for a sampled bibliography):

- a theoretical approach, in which the design problem is stated in terms of a multi-step decisional problem which cost has to be optimized with respect to a very large number of variables, some of them being combinatorial;

- a practice-oriented approach, in which the design is considered to be a progressive selection of components of the final system, but without any attention to optimality goals.

First approach is very complex and can give rise to objects which cannot be implemented. Second approach surely assures the design of implementable systems, but in general without any effective innovation.

To overcome the gap between optimality and implementation needs, the design process is here organized according to the following main lines:

(a) the design process is formulated in terms of a multi-step decisional problem, at each step of which a proposal of the new system can be defined at a proper level of accuracy;

(b) each step of the design process is dedicated to organize the best possible selection of the components of the new system (thus giving rise to a potential design manual) according to a formal model of the system itself, stated at the level of accuracy characterizing the current decisional step;

(c) once a decisional step gives rise to a potential design manual, i.e. to a new proposal of the system to be developed or innovated, a comparison with the design target has to be applied; in case the system proposal is unsufficient, a more accurate model is required and a consequent improvement of the design manual is necessary (thus starting a new innovation step).

In order to describe the approach here proposed, the paper is organized as follows. The second Section will present a potential formulation of the design problem, stated in termms of mathematical optimization: the aim is to show the problem complexity as well as to outline main ideas for solving said problem, namely the so called "innovation loop". The third Section will describe the proposal of new organization of the design process, based on the concept of "innovation loop", thus emphasizing the organizational needs for an efficient management of the design process.

Assume, for sake of simplicity, that the expected design manual consists of : 
- a description of the new final product in terms of the sequence of manufacturing operations required for its completion, and

- a description of the innovated manufacturing system in terms of set of machining centers and their aggregation into manufacturing cells.

According to this assumption (which defines the expected final level of accuracy), a mathematical formulation of a simplified design process is stated as follows (Kusiak, 1993 and Villa, 1991).

Given a new final product to be designed, assume that there exists a partially ordered set of manufacturing operations among which some operations can be applied in order to manufacture the considered product (the partial ordering specifies precedence relations over pairs of operations, as usual in machining). In practice, said set of operations can be viewed such as a set of alternative sequences of manufacturing operations, each one being a complete manufacturing sequence. In addition, assume that a set of $\mathrm{M}$ machining centers can be used for processing the new product, as well as the other NP products already manufactured by the systemm to be innovated. Each machining center is able to apply some of the operations above considered, and not all the available machining center are already utilized in the plant. The goal is the select the sequence of manufacturing operations and the set of machining centers required for its implementation in such a way to optimize the manufacturing costs.

With the aim of formulating the complete design problem, as above outlined, let us introduce the following notations:

$i, i=1, \ldots, N S$, index of a sequence of operations;

$j, j=1, \ldots, N O(i)$, index of an operation belonging to the sequence $i$;

$m, m=1, \ldots, N M$, index of the machining center;

$p, p=1, \ldots, N P$, index of the product already processed;

$[N P+1]$ denotes the new product to be designed and manufactured;

$A$, "sequence-to-operations" incidence matrix such that

$[A]_{i, j}=1$ if sequence $i$ includes operation $j ;=0$ otherwise;

$B$, "machine-to-operations" incidence matrix such that

$[B]_{m, j}=1$ if machine $m$ includes operation $j ;=0$ otherwise;

$M$, "product-to-machine" incidence matrix such that

$[M]_{p, m}=1$ if product $p$ is processed by machine $m ;=0$ otherwise;

The complete design problem can be stated in terms of the following optimization problem:

$\min \left[\sum_{i=1}^{N S} c_{i} X_{i}+\sum_{m=1}^{N M} c_{m} Y_{m}\right]$

subject to the following constraints: 
(a) constraint assuring that all manufacturing operations required for processing the new product, according to the sequence $i$ to be selected, can be applied by available machines:

$$
\exists(i) \Rightarrow \sum_{m=1}^{N M} \sum_{j=1}^{N O}[A]_{i, j} X_{i} \times[B]_{m, j} Y_{m}=N O(i)
$$

where $N O=\max \{N O(i)$, for all $i\}$;

(b) constraint assuring that the sequence of operations for processing the new product is as similar as possible to the ones currently applied to products already processed:

$$
\exists(p, \delta) \Rightarrow \sum_{m=1}^{N M}\left\|[M]_{p, m}-Y_{m}\right\| \leq \delta
$$

(c) constraints assuring complete production:

$$
\sum_{i=1}^{N S} X_{i}=1 ;
$$

As showed by the introduced statement, the considered design problem implies to jointly select the best possible set of machining centers able to process a new product which optimal sequence of manufacturing operations has to be concurrently selected. In industrial terms, it is well known that the resulting combinatorial optimization appears to be eccessively time-consuming.

\section{INDUSTRY-ORIENTED ORGANIZATION OF THE DESIGN PROCESS}

Owing to its complexity, it looks necessary to formulate a conceptual structure within which the above introduced joint-product-and-system design process can be modelled. The basic idea on which this conceptual structure is founded, consists of the "innovation loop".

In practice, the proposed design process organization consists of the following steps (Villa, 1997):

1. Assume to have at disposal a preliminar model of the system to be innovated, either described in terms of design specifications and requirements (zero level of resolution), or obtained at a given resolution level by previous iterations along the "innovation loop"). 
2. The design manager must identify every new function of the system under development, starting from each function already modelled at the previous resolution level, through an accurate selection of more accurate representations of the system itself.

3. To each function, as soon as identified, at least a system component has to be associated: said component should either implement the considered function or contribute to the function implementation with the best possible efficiency and effectiveness.

4. All components must then be connected together through suitable interfaces (to be selected), in such a way to define a graph of components, that means the structural model of the new system. The obtained model will be the best possible description of the system architecture and functionality.

5. Based on the graph of components, to each component and to each interface at least a design sub-problem must be associated, such to state how the most convenient component/interface parameters could be estimated.

6. For each type of design sub-problem, a proper solution procedure has to be identified, such as to allow the estimation of the system parameters at the highest possible efficiency (in industrial terms).

7. By applying the estimated parameter values, a model of the new system at the current resolution level is obtained. This model can be validated by comparing the model performance (e.g. evaluated through a simulator) with the expected design target. Depending on the result of this comparison, a further iteration along the "innovation loop" could be necessary, in case the current resolution does not allow an effective implementation of the obtained design result.

\section{OUTLINE OF AN APPLICATION}

The introduced method for organizing a design process is now applied in case of the conceptual joint design of new product and related manufacturing system.

According to considered design phases, the first step consists of defining the design target and the initial model of the basic function of the new system: to design and to manufacture new product.

To such a preliminary description of the basic function of the new system a proper low-resolution model has to be associated. The goal of the first-resolution model is to verify if a new product, for given approximated sequence of operations, can be manufactured by the existing system (otherwise, relevant innovations of the plant are required).

The results which can be obtained by the first iteration along the "innovation loop" will be the estimate of the production capacity of the manufacturing system to be designed, as well as a preliminar description of the manufacturing operations sequence. As it is quite obvious, this result does not reveal to be of any industrial interest. Then, more details are necessary. 
At a higher resolution level, attention of the designer must be focused on a better description of the system to be developed. Then, the model introduced at the second level of resolution will describe the design problem according to the following organization of parts:

1. First design sub-problem consists of the selection of the new product and the definition of its sequence of manufacturing operations, constrained to be executed by available manufacturing centers (this, in turn, is the task assigned to the second design sub-problem);

2. Second design sub-problem consists of the selection of the manufacturing centers to be included in the new department, under the constraint that said centers must be able to execute the sequence of operations required for the new products-

3. Third design sub-problem follows from the necessity of deriving coordinated solutions of the two above stated sub-problems, by implementing a suitable coordination procedure (see for example coordination methods developed within Large Scale System theory).

Evident connections exist among the three design sub-problems: they can be illustrated in a graph of design sub-problems, sketched in Figure 1.

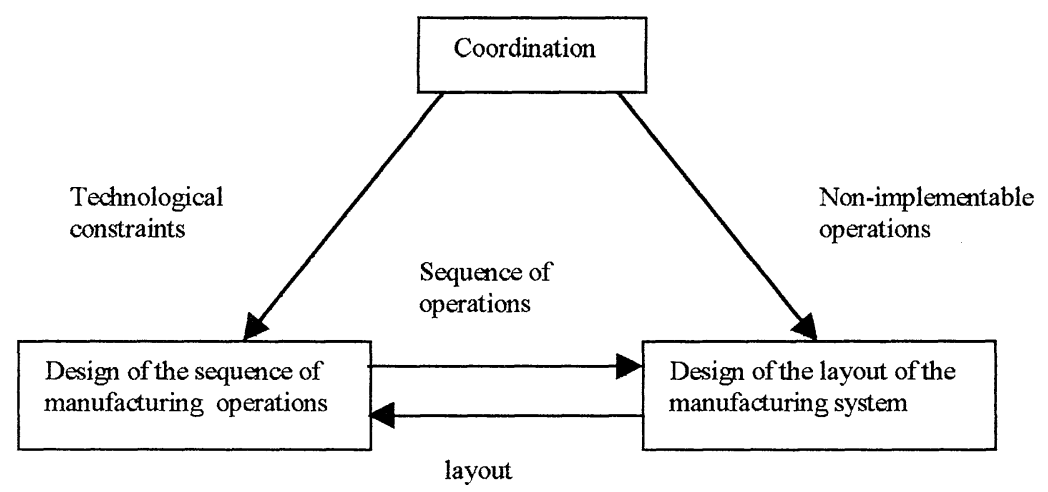

Figure 1. Graph of design sub-problems.

Intuitively this level of resolution shows that the complete design problem, stated in formal terms in the previous Section, can be decomposed into a net of three sub-problems, provided that a coordinating action is introduced. Then, a suitable organization of the design tasks (as mentioned before) is necessary if the goal is to derive an industrial design strategy.

In the present pattern of sub-problems, the coordination task is quite well defined. Infact, solution of the sub-problem devoted to organize the sequence of manufacturing operations calls for knowledge of which machining centers could be used for implementing the necessary operations. On the other hand, the 
selection of a good layout is onditioned on knowledge of all operations to be executed, as well as of the most complicated ones.

As in case of the first level of resolution, again the result of the second iteration along the "innovation loop" could be considered unsufficient, owing to a poor level of accuracy in describing both the new product and, mainly, the system to be innovated. As a consequence, a further iteration is necessary. In the following Figure 2, the reader can find a sketch of the new set of the design tasks which can be arranged at the second level of resolution, as they result from the previous identification of design steps. At this level, the modellization of the new product and related manufacturing system appears to be quite fine, but a relevant coordination is necessary.

\section{Concluding remarks}

Leaving to the reader the analysis of this second-resolution organization, a few concluding remarks are now appropriate.

The application example outlined shows the main characters of the proposed organization of a design process: (a) approaching the design task according to a gradual increase of the complexity in the new system description (thus obtaining a better and better resolution in modelling the new system); (b) at each level of resolution, decomposing the complete design problem into a set of interconnected sub-problem, but through introduction of suitable coordination tasks.

Then, the main result of the proposed approach (just applied in the organization of some industrial-founded projects) is that a right mix of decomposition and coordination can help in efficiently solving any complex industrial design problem.

Kusiak A. Ed. (1993), Concurrent Engineering - Automation, Tools, and Techniques, Wiley, New York.

Villa A. (1991), Hybrid Control Systems in Manufacturing, Gordon \& Breach, London.

Villa A., Management of Technological Innovation Processes (in italian), Clut, Turin, 1997.

Villa A., Cantamessa M. and Rossetto S. (1996), Material Handling: a Design Method (in italian), Ed. Progetto Finalizzato Trasporti Due, Consiglio Nazionale delle Ricerche, Rome.

Acknowledgments:

Present results have been developed in a research program supported by the Italian National Council of Research, under a grant of the Progetto Finalizzato Trasporti Due. 


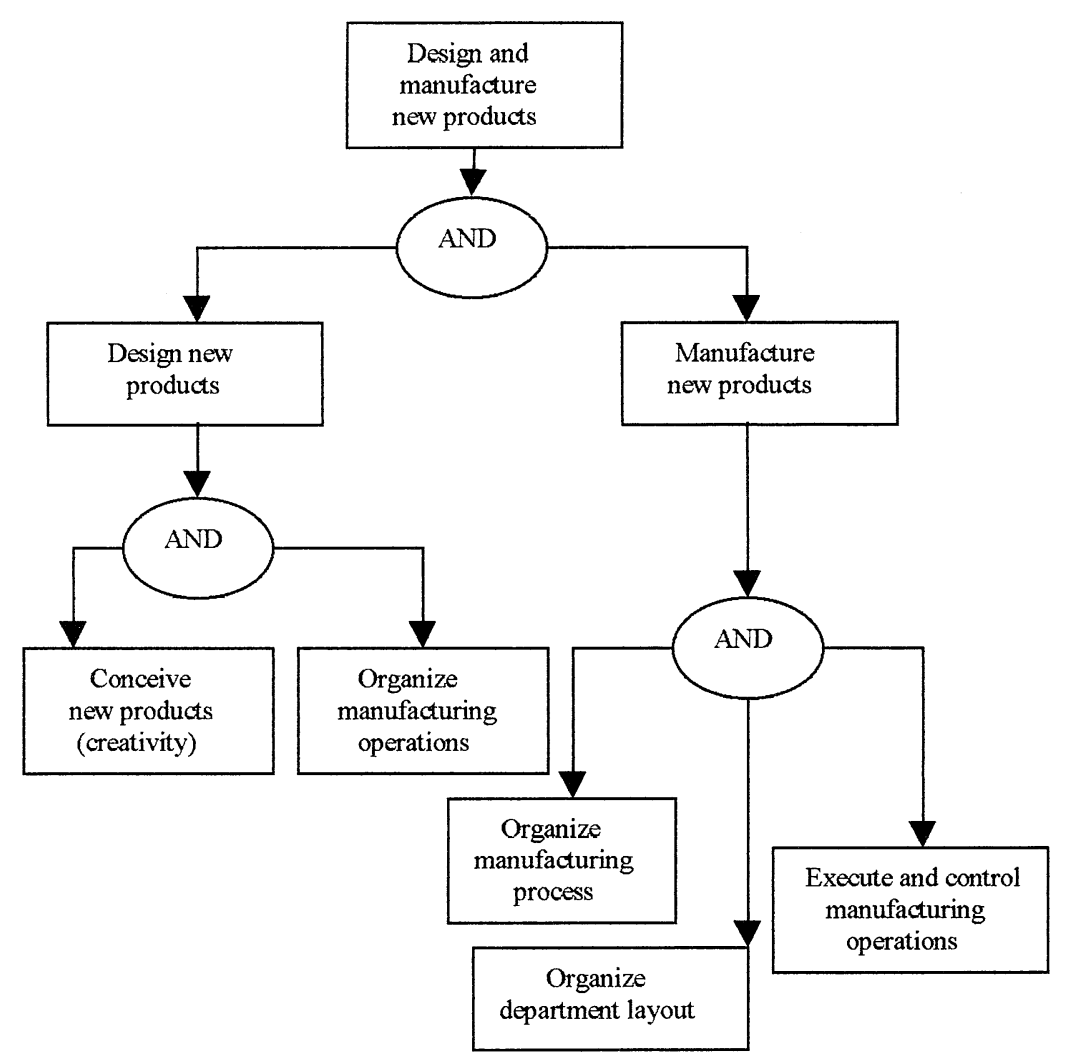

Figure 2. Function tree at the second resolution level.

\section{BIOGRAPHY}

A. Villa is full professor of production planning and control at the Technical University (Politecnico) of Turin, where he is president of the council for the laurea degree in industrial management and responsible of the Evaluation System of the university. Author of about 150 papers and 6 books, he is regional editor of the International Journal CIMS - Computer Integrated Manufacturing Systems. He currently chairs the IFAC Technical Committee on Manufacturing Modelling, Management and Control (MIM). 